\title{
Reconstrucción con colgajo anterolateral del muslo en un paciente con indicación de amputación infracondílea
}

\author{
Anterolateral flap reconstruction of the thigh in a patient \\ with indication of infracondylar amputation \\ Dr. José Barragán-Cabral,* Dr. José Antonio Morales-González,* \\ Dra. Tonatzin Loreto Rodríguez-Salazar, ${ }^{\ddagger}$ Dr. José Mauricio Barragán-García ${ }^{\S}$
}

Palabras clave: Amputación infracondílea, muñón de amputación, colgajo anterolateral de muslo,

reconstrucción.

Keywords: Infracondylar amputation, amputation stump, anterolateral thigh flap, reconstruction.

* Cirujano Plástico y Reconstructivo.

¥ Médico pasante del Servicio Social.

$\S$ Médico Interno de

Pregrado.

Hospital General de Zona (HGZ) 30 del Instituto Mexicano del Seguro Social. Mexicali, Baja California.

Los autores de este artículo no tienen conflicto de intereses que declarar.

Recibido:

20 julio 2019

Aceptado para publicar:

15 enero 2020

\section{RESUMEN}

El sistema de la arteria circunfleja femoral lateral en el muslo ha permitido, como opción reconstructiva, el desarrollo de los colgajos del músculo tensor de la fascia lata y del colgajo anterolateral de muslo; este último está compuesto de piel y tejido adiposo subcutáneo de la cara anterolateral del muslo. El colgajo puede proporcionar una extensa área cutánea con una dimensión de hasta 25 por 35 centímetros. Mostramos el caso de un hombre de 30 años, quien sufrió un accidente vial y presentó una lesión por machacamiento severo en el tercio distal del miembro inferior izquierdo. El paciente fue sometido a amputación en retropié tipo Lisfranc por el Servicio de Traumatología, pero evolucionó con necrosis e infección del colgajo cutáneo. Se valoró por Cirugía Plástica, y como medida de salvamento, se decidió realizar desbridamiento y terapia de presión negativa; una vez desarrollado un adecuado tejido de granulación, se realizó el colgajo anterolateral de muslo sensitivo e incorporando la rama anterior del nervio femorocutáneo lateral con anastomosis término-lateral de la perforante de la arteria femoral circunfleja lateral bajo microscopio, teniendo como vaso receptor el tercio distal de la arteria tibial posterior y una neurorrafia epiperineural término-terminal del nervio femoral cutáneo lateral al nervio sural. La evolución postquirúrgica fue satisfactoria, logrando una longitud anatómica adecuada, con lo que la expectativa y satisfacción del paciente con el resultado se cumplió al no tener que utilizar prótesis para caminar. Con lo que se concluye que, de esta manera, el colgajo sensitivo basado en el nervio femoral lateral es una opción de salvamento de la extremidad, ya que se puede optimizar la longitud y evitar el uso de prótesis.

\section{ABSTRACT}

The system of the lateral femoral circumflex artery in the thigh has allowed the flaps development of the tensor fasciae latae and the anterolateral thigh flap. The latter is composed of skin and subcutaneous adipose tissue of the anterolateral side of the thigh. The flap can provide an extensive cutaneous area with a dimension of up to 25 for 35 centimeters. We show the case of a 30-year-old man who suffered a road accident presenting severe crushing injury in the distal third of the left lower limb, undergoing amputation in Lisfranc-type rear foot by the trauma service, evolving with necrosis and infection of the skin flap. Assessed by the Plastic Surgery Service and as a salvage measure, it was decided to perform debridement, negative pressure therapy and once suitable granulation tissue was developed, anterolateral flap of the sensory thigh was performed incorporating the anterior branch of the lateral femoral cutaneous nerve with end-lateral anastomosis of the perforator of the lateral circumflex femoral artery under microscope, having as a recipient vessel the distal third of the posterior tibial artery and a termino-terminal epiperineural neurorrhaphy of the lateral cutaneous femoral nerve to the sural nerve. The post-surgical evolution was satisfactory, achieving an adequate anatomical length, so that the patient's expectation was fulfilled by not having to use prostheses to walk, and the patient satisfied with the result. The sensory flap based on the lateral femoral nerve is a limb salvage option, since length can be optimized and prosthesis use avoided. 


\section{INTRODUCCIÓN}

$\mathrm{Z}^{\text {I sistema de la arteria circunfleja femoral }}$ Elateral en el muslo ha permitido, como opción reconstructiva, el desarrollo de los colgajos del músculo tensor de la fascia lata y del colgajo anterolateral del muslo., ${ }^{1,2}$ Este colgajo anterolateral se compone de piel y tejido adiposo subcutáneo de la cara anterolateral del muslo, el cual puede proporcionar una extensa área cutánea cuyas dimensiones varían de 25 a 35 $\mathrm{cm}$; además, se puede lograr un colgajo sensitivo al incluir el nervio cutáneo femoral lateral (L2 y L3), conocido también como nervio cutáneo lateral del muslo o nervio femorocutáneo, que sigue un trayecto oblicuo hacia la espina iliaca anterosuperior que pasa en la profundidad del ligamento inguinal hacia el muslo, dividiéndose en los ramos anterior y posterior. ${ }^{3}$ Los ramos anteriores emergen a la superficie en un punto

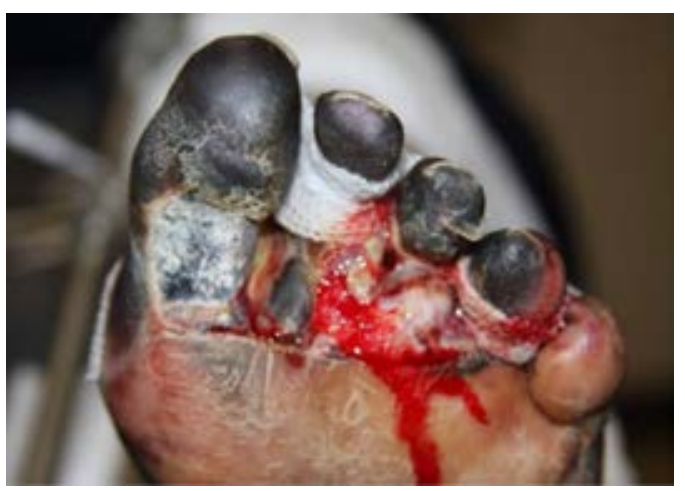

Figura 1: Necrosis de la superficie plantar de los dedos del pie izquierdo.

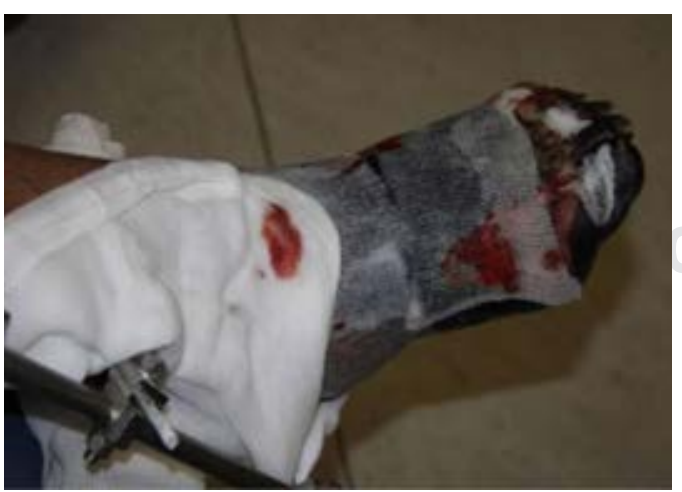

Figura 2: Necrosis del dorso del pie.

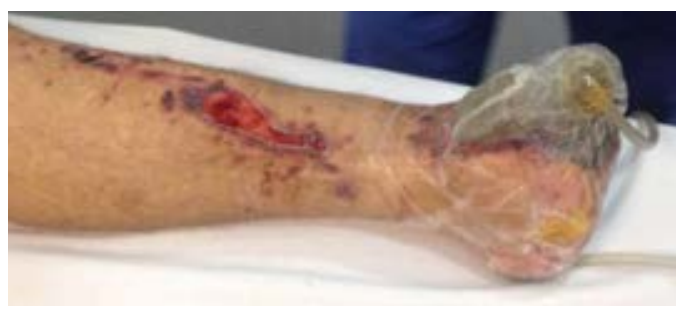

Figura 3: Terapia de presión negativa en el pie.

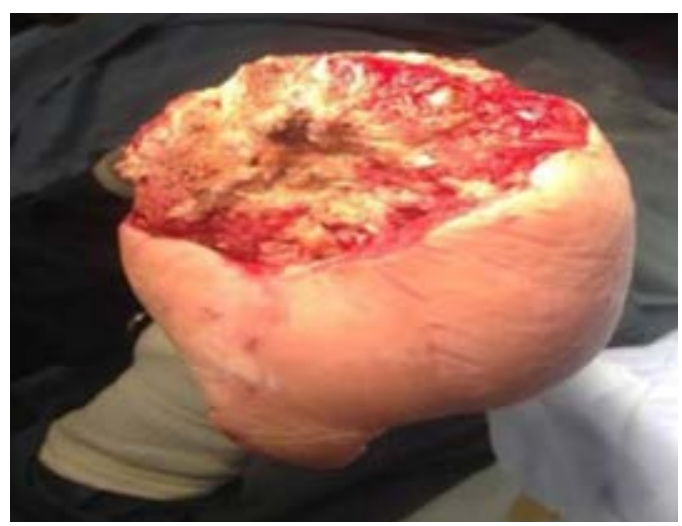

Figura 4: Tejido de granulación adecuado.

situado aproximadamente $10 \mathrm{~cm}$ distal al ligamento inguinal e inervan la piel de las porciones lateral y anterior del muslo.

En el caso clínico que se presenta a continuación, se empleó este procedimiento para evitar la amputación infracondílea, para que así pudiera tener la extremidad anatómicamente más funcional.

\section{CASO CLÍNICO}

Se trata de un hombre de 30 años de edad, quien sufrió un accidente en motocicleta y presentó una lesión por machacamiento en el tercio distal de la extremidad inferior izquierda con fractura expuesta de peroné y astrágalo; también presentaba una luxación anterior inveterada de la tibia, una lesión vascular de arterias tibiales anterior y posterior, y necrosis en el $50 \%$ del dorso y $30 \%$ de la superficie plantar del pie (Figuras 1 y 2 ).

Por esta razón, fue sometido a amputación en retropié tipo Lisfranc por parte del equipo de Traumatología; su evolución fue tórpida, 


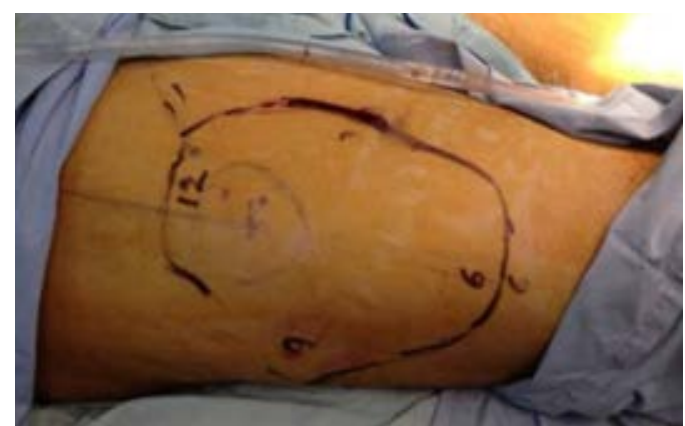

Figura 5: Marcaje del colgajo anterolateral de muslo.

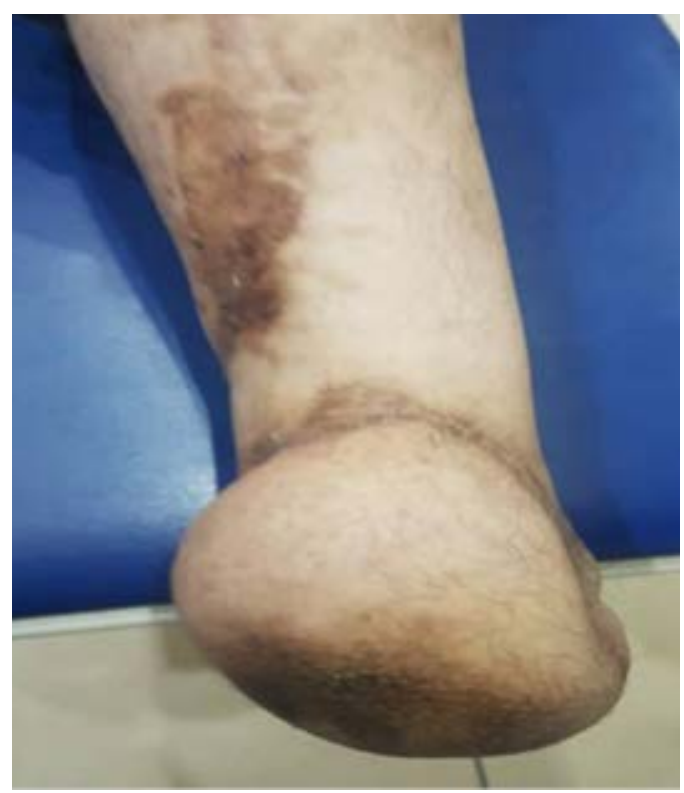

Figura 6: Postoperatorio tardío de colgajo que cubre el defecto del pie.

desarrollando en el lapso de dos semanas cambios de coloración y pérdida de flujo vascular compatible con necrosis e infección del colgajo cutáneo. Fue valorado por Cirugía Plástica, y como medida de salvamento, se decidió realizar desbridamiento y terapia de presión negativa (Figura 3). Una vez que desarrolló un tejido de granulación adecuado (Figura 4), se realizó un colgajo anterolateral del muslo sensitivo (Figuras 5 y 6), incorporando la rama anterior del nervio femorocutáneo lateral disecada en una longitud de $10 \mathrm{~cm}$. Se delimitó el colgajo anterolateral del muslo, trazando una línea desde la espina iliaca anterosuperior hasta el borde lateral superior de la patela, dibujando un círculo con un radio de $3 \mathrm{~cm}$ en el punto medio de la línea trazada. Asimismo, se utilizó Doppler para identificar otros vasos perforantes. Se levantó el colgajo y se realizó anastomosis microquirúrgica término-terminal de la arteria del colgajo a la arteria tibial anterior y anastomosis término-terminal sólo de una vena comitante. Además, se realizó una neurorrafia epiperineural término-terminal del nervio femoral cutáneo lateral al nervio sural. En el defecto del colgajo, se aplicó un injerto autólogo.

La evolución postoperatoria fue satisfactoria, puesto que se logró una longitud anatómica adecuada, con lo que la expectativa y satisfacción con el resultado se cumplió del paciente al no tener que utilizar prótesis para caminar (Figuras 7 y 8), aunque con molestias en la marcha al utilizar el calzado normal.

\section{DISCUSIÓN}

La versatilidad del colgajo anterolateral del muslo permite considerarlo como una opción para la reconstrucción de defectos complejos. ${ }^{4,5} \mathrm{En}$ nuestro caso, se logró limitar la extensión de la amputación, así como mejorar las características del colgajo al preparar el lecho receptor, utilizan-

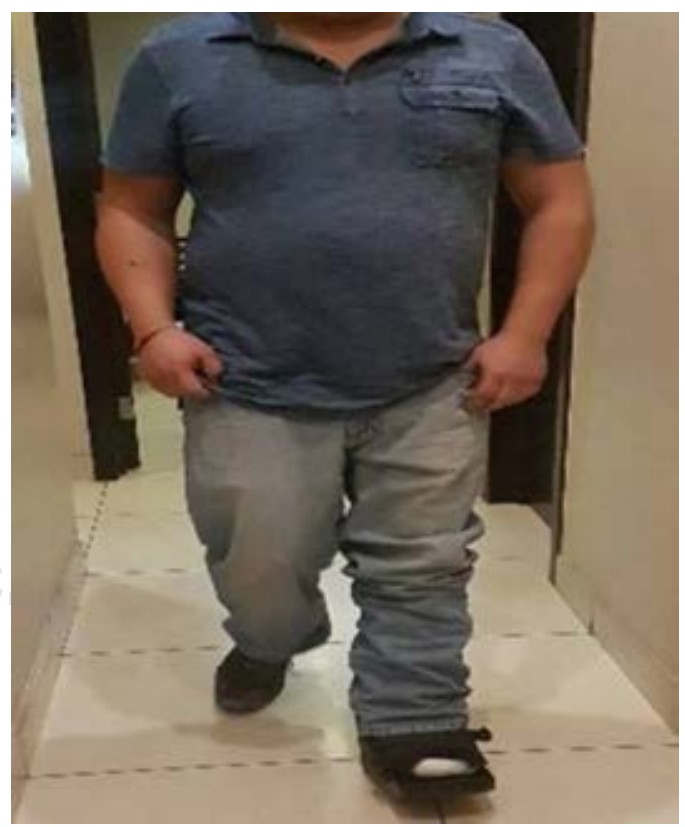

Figura 7: Deambulación sin utilizar prótesis. 


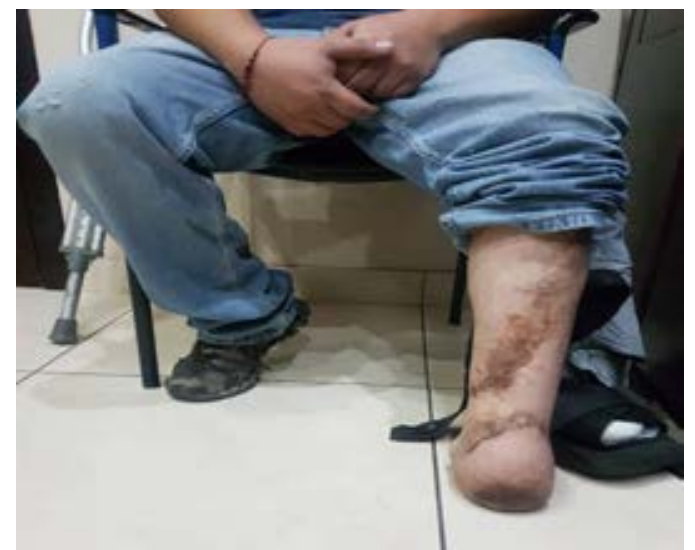

Figura 8: Resultado postoperatorio tardío.

do terapia de presión negativa e incorporando un nervio sensitivo en un paciente en edad productiva con una lesión de miembro inferior y complicaciones inherentes a la lesión.

La reconstrucción del muñón se efectuó con éxito, pues se logró una longitud anatómica adecuada, además de que la expectativa del paciente fue cumplida al no tener que utilizar prótesis para caminar.

Debido que el paciente se negó a una amputación infracondílea, hubo la necesidad de valorar la reconstrucción del muñón. Se analizó el caso y se habló con él sobre las posibilidades que tendría para volver a caminar sin la ayuda de una prótesis al reconstruir el muñón, las cuales aceptó para su reconstrucción.

\section{CONCLUSIONES}

El colgajo anterolateral del muslo sensitivo es una opción reconstructiva en pacientes jóve- nes que sufren lesiones traumáticas, ya que permite evitar el uso de prótesis y mantiene la sensibilidad, llevando a una rehabilitación adecuada. En nuestro caso, se tomó la decisión correcta para la reconstrucción del muñón, tanto funcional, estética como psicológica. La reconstrucción del muñón a este nivel permite al paciente poder caminar sólo con la ayuda de una bota ortopédica, incluso con un calzado normal. Hasta el momento, el paciente ha referido que no tiene molestias al caminar con la bota, sin embargo, al utilizar zapato existe una pequeña incomodidad debido al volumen del muñón.

Finamente, una desventaja a considerar es que la piel del colgajo no cumple con las características anatómicas de la planta del pie.

\section{REFERENCIAS}

1. Luenam S, Prugsawan K, Kosiyatrakul A, Chotanaphuti T, Sriya P. Neural anatomy of the anterolateral thigh flap. J Hand Microsurg 2016; 7 (1): 49-54.

2. Karonidis A, Bouloumpasis S, Apostolou K, Tsoutsos D. The use of the ALT flap and lateral femoral cutaneous nerve for the reconstruction of carpal soft tissue and ulnar nerve defects: a case report. J Hand Microsurg 2016; 7 (1): 182-186.

3. Valdatta L, Tuinder S, Buoro M, Thione A, Faga A, Putz R. Lateral circumflex femoral arterial system and perforators of the anterolateral thigh flap: an anatomic study. Ann Plast Surg 2002; 49: 145-150.

4. Priego BR, Trejo CD, Haddad TJL, Caracheo RR, Villanueva MA. Colgajo lateral de muslo: aplicaciones clínicas. Cir Plast 2012; 22 (3): 126-133.

5. Chana JS, Wei FC. A review of the advantages of the anterolateral thigh flap in head and neck reconstruction. Br J Plast Surg 2004; 57: 603-609.

\author{
Correspondencia: \\ Dr. José Barragán-Cabral \\ E-mail: drjosebarragan@yahoo.com
}

